\title{
15. Artificial Neural Networks, Postdigital Infrastructures and the Politics of Temporality
}

\author{
Andreas Sudmann
}

\begin{abstract}
This essay examines the infrastructures and temporalities of modern AI technology based on artificial neural networks (ANN) and aims to contribute to a more substantial understanding of its political challenges. In order to unlock the different temporalities of ANN, a theoretical framework for the relationship of media and infrastructures is suggested that also might help to distinguish between the different levels of analysis related to specific steps and aspects of the machine learning process (the collection and production of learning data, the training of AI models etc.). An important reference point for the following considerations is ethnographic research conducted at TwentyBN, ${ }^{1}$ a Toronto and Berlin based AI company specialized in ANN and computer vision that just recently developed an app for the fitness market.
\end{abstract}

Keywords: artificial intelligence, machine learning, artificial neural networks, computer vision, science and technology studies, media-ethnography

Since around 2012, machine learning based on artificial neural networks (ANN) has become the dominant paradigm of artificial intelligence (AI) research and increasingly determines the technological and infrastructural conditions of contemporary computer culture. Whether new developments in the field of text, speech, or image recognition, whether concrete use cases such as self-driving cars, virtual avatars, or medical diagnostic systems, the list of innovations that involves ANN-based machine learning is extremely

Volmar, A. and K. Stine (eds.), Media Infrastructures and the Politics of Digital Time: Essays on Hardwired Temporalities. Amsterdam: Amsterdam University Press, 2021 DOI 10.5117/9789463727426_CH15 
long. Although some scholars emphasize the epistemological limits of ANN, it is nevertheless surprising to see how, since very recently, they became such a powerful technology of prediction, capable of calculating and mastering the uncertainties and fuzziness of future events. ${ }^{2}$

The status of ANN as predictive systems already demonstrates the fundamental importance of investigating this technology in terms of its temporal dimensions. From the perspective of media studies, which in part also sees itself as a decidedly critical discipline, the particular relevance of this focus lies not only in exploring the epistemological potential of this specific approach in AI. Rather, it is the central aim of this essay to show how a deeper investigation of the infrastructures and temporalities of modern ANN-driven AI technology also contributes to a more substantial discussion of its political challenges.

Like basically all AI technologies since the 1950s, ANN have been negotiated mostly in the mode of utopian discourse, from Frank Rosenblatt's Perceptron model ${ }^{3}$ to Google's Neural Machine Translation. The term utopia, in the sense of its etymological origin, refers to a non-place, in temporal terms typically to the not-yet-existent of an unknown future. Indeed, the current advances in that particular field of AI do once again fuel the speculative notion of a so-called "technological singularity" as the very event after which all future development of machine intelligence will no longer be predictable or comprehensible to humans (while also signifying the very date when machines will be superior to humans in most, if not all, core areas of human expertise). ${ }^{4}$ Michel Serres has argued that the concept of time in itself articulates the dialectics of predictability and unpredictability. ${ }^{5}$ However, in the case of ANN, we are confronted with a specific dialectic or paradox of temporal orders that simultaneously resonates with the speculative dystopian (rather than eutopian) character connected to current visions of an artificial general intelligence: how the accelerated development of a predictive technology might turn into something — at least for humansfundamentally unpredictable and inaccessible.

But these characteristics are not concerned merely with future scenarios because ANN are already perceived fundamentally as black boxes not understandable or at least only partially understandable by humans. ${ }^{6}$ Their black-box status encompasses many aspects, not only the opacity of the ANN itself or its specific medial learning operations, or the tremendous speed of micro-temporalities connected to AI technologies and their respective decision-making processes that we can understand as submedial forms operating below the threshold of human perception, ${ }^{7}$ but also the complex industrial practices and environments that generate and form 
the technological processes of modern AI. In other words, the event of a technical singularity is an escalation of what is already being discussed as one of the core problems of ANN technology: its fundamental inaccessibility. Obviously, such an event (if it ever becomes a reality) will result in enormous distortions, not least in political terms. Against this very background, one must take such an event as seriously as possible, even if, from today's perspective, it seems a scenario of a still distant future or unlikely in principle. Nevertheless, it is not very helpful to constantly speculate about the political or social consequences of a technology's future if we have just started to understand the current situation of AI in general and ANN in particular, both theoretically and historically. Even if the present and its temporality is always exactly what is already over when we talk about it. ${ }^{8}$ Accordingly, the aim of this essay is to explore the relation between infrastructures and temporalities with regard to ANN-based machine learning, specifically to provide a substantial basis for future political discussions of this technology.

A central aspect of ANN in political terms, particularly concerning its present form, is that we are dealing with a powerful technology of optimization and automation, which in numerous areas not only expands the respective capabilities of humans, but also clearly outperforms them. And it is worth highlighting that such ANN systems do not just master domain-specific tasks faster or more efficiently than humans. Instead, in many areas, such as image recognition, they also outperform humans in qualitative sense. However, as many scholars working in the transdisciplinary field of science and technology studies have argued, it is problematic to limit the political-epistemological relationship to the binary relationship of humans and machines or humans and technology. While it is important to stress that current machine learning technologies are still very much dependent on the activities of human beings and human labor, we also have to acknowledge that we are dealing with complex infrastructures and chains of operations, where humans, things, practices, and media are inseparably connected.

\section{Theoretical Approaches to Media and Infrastructures}

But how do we make sense of these different layers of temporalities involved in the industrial and scientific infrastructures of current AI? And how deep do we have to explore all the elements that constitute and shape these smart technologies? The answer to this question can only be given if we are willing to investigate the specifics of ANN technologies and try to understand the complexity of the technology in terms of its manifold 
temporal dimensions, at least on a conceptual level. Obviously, there is no lack of potentially useful approaches: Scholars working in the field of media archaeology, science and technology studies, historical epistemology, critical code studies, platforms studies, etc., have provided many accounts for the critical understanding of our data-driven algorithmic culture, also and especially with regard to the nexus of infrastructures, media, and temporality. ${ }^{9}$ And yet we have to acknowledge that studies in this specific area of research (as with media studies) have neglected machine learning in general and ANN in particular as an important field of research for a very long time. In the course of the current AI boom and especially since the spectacular victory of DeepMind's AlphaGo, the situation has changed significantly: AI has already become the prevalent topic in every field of the humanities and sciences. Nevertheless, it has to be said that the debate on ANN or machine learning in fields such as media studies, and cultural studies more generally, is still in its infancy. But what does it mean to approach the infrastructures and temporalities of AI and ANN, especially from a media studies perspective? And even more central in theoretical terms, what is the difference between infrastructures and media (if there is any) as key concepts for understanding AI technologies?

As one might imagine, there is not easy answer to this question. And yet, while infrastructures and media share certain characteristics-for example, that they refer to the cultural productivity of (partly) invisible operations and structure $^{10}$ - I would claim that a key difference between the terms is that "infrastructure" is a concept more useful for describing the arrangement or organization of (different) media, while, in turn, media are the heterogenous entities that constitute and contribute to shaping certain infrastructures in the first place. This consideration is by no means at odds with the idea that an infrastructural or logistic dimension is central to many diverse forms of "old" and "new" media. ${ }^{11}$ Still, we need a term for the arrangement and organization of different media, especially with regard to systemic and rule-based aspects of their stabilization, as well as with regard to the interoperability and connectivity of and associated with media and their related practices. And it seems to me that the term infrastructure is suitable for precisely this purpose.

In the following, I would like to demonstrate how the distinction between media and infrastructure is also helpful for understanding the different temporal logics and dimensions of AI technologies: For example, although the differences between media as input data play an important role in choosing and shaping a particular AI infrastructure for mastering an individual learning task, we also have to take into account that media differences can be decisive for developing advanced forms of AI. For this very reason, it is 
important to deal with the specifics of the relationship between media and infrastructures as they pertain to AI technologies.

However, as I outline in this chapter, ANN systems are characterized by a rather general temporal characteristic that is of great relevance both transhistorically and across different applications and specific infrastructures, and as such challenges the perception of ANN as a recent manifestation of digital change.

An important reference point for the following observations and considerations is my current ethnographic research at TwentyBN, ${ }^{12}$ a Toronto and Berlin based AI company specialized in Deep Learning solutions and computer vision that just recently developed an app for the fitness market.

\section{Big Data, Machine Learning, Crowdworkers, and the Politics of Temporality}

To unlock the different temporalities of modern ANN and specifically to understand the political dimension of their media and infrastructures, we first of all have to distinguish between different levels of analysis related to specific steps and aspects of the machine learning process. One important perspective in this regard is to shed light on the level of collecting and producing the large amounts of data necessary for training a machine to master a certain learning task. For example, in the case of so-called supervised machine learning, it is not enough simply to have access to large data sets; it is also necessary to have pairs of corresponding inputs and outputs as training data. A profound challenge here is that in many use cases, learning data in accurately labeled form is not available, and producing it can be a very time-consuming and costly working process. To address this problem, AI companies like TwentyBN specializing in ANN technologies hire legions of crowdworkers through platforms such as Amazon Mechanical Turk or CrowdFlower, responsible for labeling and producing the learning data. A general and and economically productive advantage of crowdsourcing in temporal terms is that instead of having a few employees working on monotonous, repetitive tasks again and again, such activities can be distributed more or less in parallel to hundreds or thousands of workers, thus saving a massive amount of time. ${ }^{13}$

One of the downsides of this form of work in late capitalism is that crowdworkers are typically poorly paid, which in turn regularly has a negative impact on the work they are engaged for. For their labor to pay off at least to some extent, they must complete their specific tasks as quickly 
as possible. Hence, to fulfill the task of producing and labeling data most efficiently, it happens quite often that they develop subversive strategies to earn more money within a certain time frame. Unsurprisingly, such practices of "cheating" do have serious consequences; in essence, it means that the algorithms are trained with corrupted data. As a result, AI companies like TwentyBN usually cannot use the data produced by crowdworkers directly; instead, they are forced to monitor and evaluate its quality using test algorithms or manual inspection. As the activities of crowdworkers illustrate, ANN systems and their specific infrastructures do have profound effects on the temporalities of lived experience, they not only exert pressures of conformity or standardization but also lead to strategies for avoiding the temporal regimes of a cognitive capitalism and its infrastructures now increasingly shaped by data-driven machine learning.

The creation of appropriate learning data can take months or even years, depending on the specific purpose of the learning data and AI model. The training and testing of an AI model is also very time-consuming. For high-end AI applications, such as advanced machine vision systems, training involves not only thousands but typically even millions of such cycles or epochs, while other less complex classification tasks, such as distinguishing simple geometrical forms (as in Rosenblatt's perceptron model), might only demand a few hundred training cycles. Hence, the temporal span of a training process can also vary a lot, from a few hours to several weeks. More generally, how fast an ANN can be trained for a certain problem depends on number of different parameters, including the quantity and quality of the training data, the specific architecture of the ANN, and the hardware resources available. For these very reasons, it is crucial to understand the specific characteristics of AI infrastructures that make use of ANN and other technologies of machine learning.

\section{Real-Time Capability and the Temporal Challenges of Cognitive Capitalism}

Another important point to consider regarding the temporal dimensions of AI infrastructures is that many intelligent systems must be capable of operating in real-time.

When for example TwentyBN recently developed its fitness app, it was particularly important to the company that their AI system is capable of providing immediate feedback to the users whenever they perform a certain exercise like jumping jacks or push-ups incorrectly. 
And also especially in sensible application areas, such as self-driving cars, ANN-based AI models involve strict latency requirements and "demand lightning-fast deep learning inference, usually within tens of milseconds for each sample."'14

Many modern AI technologies are therefore media configurations, which should or must guarantee a certain degree of responsiveness (Rechtzeitigkeit), which means they have to operate with regard to a varying ratio of processing speeds and a time window to be adhered to by these processes. ${ }^{15}$

While forms of "inference acceleration" have significantly improved over the last few years, also due to new hardware architectures like Google's TPUs, there is a further serious problem of temporality to consider here. For example, ANN models (like any other machine learning model) are based on the assumption that there are no "distributional shifts in the input and output data over time. ${ }^{{ }^{16}}$ Machine learning and in particular ANN systems work so well, regardless of the specific prediction task, because the world, i.e., the structure of data a statistical model of AI can learn, usually does not change quickly or with hard transitions but usually slowly and smoothly. ${ }^{17}$ And yet, the important assumption of ANN and other machine learning models that the distribution of input and output data remains more or less stable over time does have its specific limits, especially if we think about application areas like information security, "where fast-paced evolution of the underlying data generating mechanism is a norm (in the case of security, it is because both players, the defender and the adversary, are constantly striving to outmatch his opponent by changing his own strategies, thus exploiting the opponent's unguarded vulnerabilities)." ${ }^{m 8}$

Nevertheless, the basic principle of the industrial use of modern technology as a whole is to ensure that technical problems can be dealt with quickly and flexibly, for example, in the field of AI with regard to the much-discussed problems of bias structures. However, this does not always work as smoothly as desired. When in 2016 Microsoft presented its chatbot Tay to the public, the company had to shut down the system only after sixteen hours. Users quickly hacked the system by training it for racist, anti-Semitic, and sexist articulations, which unsurprisingly led to quite a large public controversy. But even if the example of Tay illustrates some serious problems and limits of current machine learning systems, we should not forget that at least with regard to machines, problematic bias structures can be easily corrected, or at least there is the option to simply shut down systems that do not work properly. And this is obviously different with regard to human beings.

However, within current academic discourses of philosophy, social science, and cultural studies, temporal aspects seem to play a less important 
role when we discuss the specific potentials of AI. This may be because AI itself is overdetermined by a rhetoric of progress emphasizing efficiency and flexibility in accordance with the neoliberal logic of the temporal regimes of late capitalism.

While this form of subjugation to neoliberal orders of time is typically rightly questioned by scholars across different fields, in the humanities and beyond, we should also keep an eye on the potentials of AI, even if the technology and its temporal order as a whole or in part are never neutral, serve neoliberal interests, or take on a questionable and teleological logic of progress. To give just one of many examples, if traffic will become more and more dominated by autonomous vehicles in the future, or if road traffic will be regulated by modern AI systems, this may be seen as a problematic affirmation of neoliberal values of optimization and efficiency because the intention is not simply to reduce traffic jams but also to create the possibility of coping with greater traffic loads, which in turn represents a serious ecological problem. On the other hand, there is at least some evidence that self-driving cars will significantly reduce the frequency of traffic accidents, simply because they are faster and better able than humans to recognize dangerous situations as such. ${ }^{19} \mathrm{~A}$ critique of $\mathrm{AI}$, which in particular focuses on aspects of temporality, should therefore take such ambivalences of technology into account and discuss them in a differentiated way.

\section{The Potential of ANN to Understand and Identify the "New"}

In any case, it is interesting to note that the critique of AI often includes a critique of the temporality of these systems. For example, the fact that machine learning technology inevitably uses past data to predict the future has often led to skepticism from scholars, claiming that data-driven machine learning systems based on ANN are inherently conservative and incapable of producing something genuinely new. ${ }^{20}$ Indeed, one could argue that recent discussions about algorithmic biases underpin this critical perspective. Likewise, many of us know from our everyday experience that it can be quite boring when, for example, recommender systems simply suggest titles similar to the music we already like. It might be also fair to say that we don't necessarily have to make the algorithms responsible for this: If we have the impression that an AI-powered system is only suggesting music we already know or like, we could also take this as an invitation to question the limitations of our taste in music. But that's not the point I'm primarily concerned with. Instead, I would like to question the criticism I have just presented for two other reasons. 
First, doubts seem justified as to whether ANN-based systems are really incapable of producing something new in the emphatic sense. Isn't the example of DeepMind's AlphaGo a powerful reminder that we can witness an AI model operating with moves that are perceived as innovative and surprising by the global community of Go players? Of course, prima facie it seems legitimate to argue that, according to such a perspective, one does not just take the rhetoric and PR strategies of the AI industry for granted, but also carry out a questionable anthropomorphizing and mystification of technical systems that in their core consists of statistical or computational processes. Nevertheless, it might be advisable to distinguish between Genesis and Geltung here. Similar to Turing's argumentation in the 1950 s regarding the justification of his famous test, one could argue that what matters is less whether an AI system really is creative or if it de facto has the potential in itself to create something genuinely new. Rather, what counts is if the system manages to appear to be creative or innovative.

Second, the question would be if the fetishization of AI, which has often been criticized, does not provoke another form of fetishization, namely the fetishization of an anthropocentric figure of the emphatically new. ${ }^{21}$ All "human" achievements, whether in art or science, are in fact based on complex and preceding mediators, i.e., on already existing technologies, media, infrastructures, communication processes, and forms of knowledge. Obviously, we apply different standards to machines than to people. But why? Do we have to face the fact that a post-anthropocentric view of AI is so difficult to accept, not only because we are not willing but also because we are simply unable to do so? I do not suggest we have to answer that question in the affirmative. Rather, the point is that one must be aware of the specific paradoxes when discussing anthropocentric concepts such as learning, knowledge, or the "new" in relation to intelligent machines.

However, the key challenges that AI systems are currently confronted with are indeed less about being able to identify or generate something completely new than about being able to take account of the temporal dimension as well as the specific context of data.

\section{Recurrent Neural Networks and the Temporal Significance of Media Differences}

It is important to acknowledge that also in this very area ANN have made significant progress in the last ten years. A crucial factor here was the advanced development of so-called recurrent neural networks (RNN). A 
key characteristic of RNN is their ability to identify patterns in sequences of data, e.g., for learning tasks such as text understanding, speech recognition, and even stock market developments. To do so, RNN need to fulfill the same task for each of the sequence's elements, while the output depends not only on the current input but also on the previous computations. In other words, RNN have a kind of memory function (internal state) that allows information to persist. Theoretically, RNN can process data sequences of infinite length, but in practice they are constrained so that they can look back only a few steps. Although the gradients of RNN can quite easily be computed, they are difficult to train due to their nonlinear iterative characteristics. According to Ilya Sutskever,

A small change to an iterative process can compound and result in very large effects many iterations later; this is known colloquially as "the butterfly-effect." The implication is that in an RNN, the derivative of the loss function at one time can be exponentially large with respect to the hidden activations at a much earlier time. Thus, the loss function is very sensitive to small changes, so it becomes effectively discontinuous. ${ }^{22}$

However, these limitations and difficulties do not change the fact that the leaps in performance in the area of speech recognition, such as with Alexa, and Siri, or in the field of machine translation have been considerable since around 2009.

Recurrent neural networks have also made significant progress in the field of computer vision. In 2016, I had the honor of introducing what might be considered as the most important ANN innovation since the famous ImageNet-Paper by Krizeshvsky et al $^{23}$ in an article for the German newspaper Frankfurter Allgemeine Zeitung, ${ }^{24}$ where I described how for the first time in the history of information technology, TwentyBN developed an ANN system to automatically recognize hand gestures and basic (and yet complex) activities (even before Baidu, Facebook, and Google were able to master this fundamental AI problem). And, indeed, the infrastructure (or the media) that enabled this computer vision technology could hardly be more relevant from a media studies perspective: For the first time and in contrast to most approaches in this field of AI research up to this point (which typically used photographs or still images for computer vision tasks), TwentyBN's model has been trained with hundreds of thousands of short video clips, showing different kinds of (basic) activities, such as opening objects, throwing and catching something, or stack things. ${ }^{25}$ The implications of this approach for our thinking about infrastructures, media, temporality, 
and their relations are very significant: What the system developed by TwentyBN implicitly demonstrates is that already the sheer media difference between time-based and non-time-based media seems to be an essential precondition for the development of an advanced AI. Accordingly, since 2017, we are confronted with new epistemological condition of our global computer culture, namely that we witness the emergence of an AI that is already capable of understanding basic forms of common-sense knowledge, i.e., perceiving and "understanding" the world in ways similar to human beings. ${ }^{26}$

And - hardly less relevant—we also have to consider that the specific technological potential of media difference to allow such advanced forms of machine intelligence exists - at least in a certain sense-outside the realm of history, culture, and ideology. On the one hand, the specific content and meaning of the videos shown to the system is less decisive for the learning operation than the basic fact that it is trained with moving images at all. On the other hand, the "content" of the model matters insofar as the system has to be trained with a great variety of gestures and actions so that it is capable of generalizing well. Nevertheless, the learning system also perceives and processes every input similarly, without a deeper sense of meaning, the gesture of a Hitler salute (Hitlergru $\beta$ ) not being different from the activity of turning a bottle, stacking books, or doing jumping jacks. Hence, ANN-based computer vision takes place within an infrastructural arrangement in which the broader cultural, social, and historical contexts of the learning material play no significant role in terms of their technological operations. Functionally decisive is the diversity of the displayed material in itself, not its specific and semantically charged composition. ${ }^{27}$

As the example outlined above shows, it is very important to focus on the specific, inherent temporalities of learning algorithms, their Eigenzeitlichkeit, which at least partially retain autonomy and contingency over those temporal regimes that characterize the commercial and scientific infrastructures of machine learning technologies as a whole-such as the inscription of history or the specific temporal logic of how machine learning tasks are organized as industrial or scientific processes.

Against this background, in view of their principal indifference to content and meaning, ANN systems are a media technology that is not fundamentally different from the gramophone or photography as technologies of the nineteenth century or from the digital computer as a technology of the twentieth century. And yet ANN turn the time relations of information technology upside down. 


\section{Massive Parallelism as a Postdigital Configuration of Future Information Technology}

An important prerequisite for the implementation of a model such as that developed by TwentyBN, however, is the performance of the modern computer hardware on which the networks are trained, namely graphics processing units (GPUs) ${ }^{28}$ What distinguishes the temporality of ANN both generally and transhistorically is their massive parallelism. At the end of the 1980s, Friedrich Kittler wrote:

Today's artificial intelligences run faster, more parallel, but not fundamentally different from those who "follow the principle of the Universal Discrete Machine ... With it, the media system is closed. Storage and transmission media both merge into a principle circuit that can simulate all other information machines simply because it stores, transmits and calculates in each individual program loop. ${ }^{29}$

In contrast to Kittler's perspective, I would like to argue that ANN systems, especially because of the parallelism of their information processing, do indeed represent a significant break with the logic of digital computers of the von Neumann architecture and that they actually stand for the emergence of a post-digital computer culture avant la letter. ${ }^{30}$ In fact, neural networks, whether artificial or "natural," are in at least two respects a counter-model to the way digital computers function according to von Neumann's serially organized architecture. First, since a single artificial neuron is usually either active or not, in this respect it usually functions according to binary logic, such as the switching states of a digital computer. The weighting of the activity between the neurons, i.e., the strength of their connections, is however mostly represented by floating point numbers (positive and negative) in neural networks. And this representation is so finely structured that the corresponding values can be understood as quasi-analog. As media of information transmission, ANN do not operate using binary units, such as 0 and 1 , but in an almost analog form (even if the analog values are still based on a digital substrate).

Second, it must be emphasized that the masses of interconnected neurons, activated by an input, fire together simultaneously or in parallel, thus ultimately forming a complex emergent system that abolishes the discreetness of the elements it consists of (the layers of neurons and their connection). This extreme or massive parallelism of information processing can indeed count as the essential characteristic of ANN, distinguishing it from the von 
Neumann architecture of classical digital computers. Due to the described properties, an ANN is therefore a blurred system (Unschärfesystem) with probabilistic results, whose operations can be described rather as analog than digital..$^{11}$ Whether ANN is primarily implemented as software, as it is at present, or increasingly as hardware, as it may be in the future, is irrelevant for its characterization as a post-digital information technology. Whatever future processor technologies modeled on neural networks will look like concretely, they share the basic characteristics that already position ANN as a "software medium." Anyone who simply understands the current developments of ANN, particularly in terms of its political dimensions, as a further expression of the digital revolution or the digitization of culture is in fact using the wrong category - at least in part.

\section{Conclusion}

In recent decades, the evolution of computers and the processes of digitization have been extensively investigated with regard to their temporal conditions, implications, and effects. Again it was Friedrich Kittler who argued that the computer, as a universal medium that can scan and simulate all other media, is in principle the end of media history..$^{32}$ Furthermore, several scholars have conceptualized the temporal logics of digitization as a transformation process of simultanization. The current renaissance of ANN now once again draws attention to the history of sub-symbolic information processing and its specific logics of temporality. With a few notable exceptions, the field of media and cultural studies has until very recently payed little attention to this. Paul Virilio, for example, has written about AI-driven vision machines that replace human perception, machines that look back at us and observe us, but despite its interest in the dromological view on temporality, he did not realize the enormous temporal implications of the connectionist AI paradigm that is now on the rise again. ${ }^{33}$

But what exactly has changed? One could assume that thanks to the massive parallelism of ANN-based machine learning we are confronted with a new dimension or quality of technological acceleration and thus have reason to believe that the event of a technological singularity might indeed not be that far away. But this remains to be seen. Obviously, the era of digital computers has not yet ended. Nevertheless, it is important to think about the changed conditions of a sub-symbolic computer culture, which we may no longer be able to adequately describe as digital. 
In contrast to the world of cultural imaginations, where immortality and death, series and events, infinity and finiteness, constitute a symbolic, interwoven network and thus a mysterious world sui generis, the question of AI infrastructures and their temporalities as a techno-political condition of the present almost adheres to something soothingly down-to-earth: be it as a view of the material preconditions of metaphysical speculations or as a more precise determination of the organizational relationship of media in relation to their environments. Perhaps today more than ever, to preserve the utopian potential of AI technology, we must lead it out of the mode of its speculative discourse.

\section{Notes}

1. "Media and Infrastructures of Artificial Intelligence: Computer Vision, Transfer Learning, and Artificial Neural Networks as Black Box.", funded by the German Research Foundation since 2019.

2. See Ian Goodfellow, Yoshua Bengio, and Aaron Courville, Deep Learning (Cambridge, MA: MIT Press, 2015).

3. See Frank Rosenblatt, "The Perceptron: A Probabilistic Model for Information Storage and Organization in the Brain," Psychological Review 65, no. 6 (1958): 386-408. https://doi.org/10.1037/hoo42519.

4. See Vernor Vinge, "The Coming Technological Singularity: How to Survive in the Post-Human Era," in NASA Lewis Research Center, Vision 21: Interdisciplinary Science and Engineering in the Era of Cyberspace, NASA Conference Publication CP-10129 (San Diego, CA: NASA, 1993), 11-22; Nick Bostrom, Superintelligence: Paths, Dangers, Strategies (Oxford: Oxford University Press, 2014).

5. See Michel Serres and Bruno Latour, Conversations on Science, Culture, and Time, trans. Roxanne Lapidus (Ann Arbor: University of Michigan Press, 1995).

6. Andreas Sudmann, "Deep Learning als dokumentarische Praxis," Sprache und Literatur 48, no. 2 (2017): 155-170.

7. Wolfgang Ernst, Digital Memory and the Archive, ed. Jussi Parikka (Minneapolis: University of Minnesota Press, 2013); Mark B. N. Hansen, Feed-forward: On the Future of Twenty-First-Century Media (Chicago: University of Chicago Press, 2015); and Shintaro Miyazaki, "Algorhythmics: Understanding Micro-Temporality in Computational Cultures," Computational Culture 2 (2012), http://computationalculture.net/algorhythmics-understanding-micro-temporality-in-computational-cultures/.

8. Jacques Derrida, Marges de la Philosophie (Paris: Minuit, 1972).

9. See Lisa Parks and Nicole Starosielski, eds., Signal Traffic: Critical Studies of Media Infrastructures (Urbana: University of Illinois Press, 2015); Gabri- 
ele Schabacher, "Medium Infrastruktur: Trajektorien soziotechnischer Netzwerke in der ANT," Zeitschrift für Medien- und Kulturforschung 2 (2013): 129-148; Axel Volmar, ed., Zeitkritische Medien (Berlin: Kadmos, 2009).

10. Susan Leigh Star, "The Ethnography of Infrastructure," American Behavioral Scientist 43, no. 3 (1999): 380.

11. See John Durham Peters, The Marvelous Clouds: Toward a Philosophy of Elemental Media (Chicago: University of Chicago Press, 2015).

12. "Media and Infrastructures of Artificial Intelligence: Computer Vision, Transfer Learning, and Artificial Neural Networks as Black Box," funded by the German Research Foundation since 2019.

13. Nevertheless, as I have learned during my ethnographic study so far, there are many crowdworkers who take on these microtasks repeatedly, sometimes even for years.

14. Yanchen Wang, "Deep Learning in Real Time-Inference Acceleration and Continuous Training." Medium.com, 2017. https://medium.com/syncedreview/deep-learning-in-real-time-inference-acceleration-and-continuous-training-17dac9438bob.

15. Uwe Brinkschulte and Heinz Wörn, Echtzeitsysteme. Grundlagen, Funktionsweisen, Anwendungen (Berlin: Springer, 2005); Volmar, Zeitkritische Medien, 10,18 .

16. Wang, "Deep Learning in Real Time."

17. Ethem Alpaydin, Machine Learning. The New AI (Cambridge, MA: MIT Press, 2016).

18. Wang, "Deep Learning in Real Time."

19. See Sprenger in this volume.

20. See, e.g., Matteo Pasquinelli, "Machines that Morph Logic: Neural Networks and the Distorted Automation of Intelligence as Statistical Inference," Glass Bead Journal, Site 1, Logic Gate: The Politics of the Artifactual Mind (2017), https://www.glass-bead.org/article/machines-that-morph-logic/?lang=enview.

21. See also Marvin Minsky's remarks from the early 1980 os on the creativity of machines and how in comparison human creativity tends to be overrated and mystified whenever people discuss the limits of AI. Marvin Minsky, "Why People Think Computers Can't," AI Magazine 3, no. 6 (1982), 3-15.

22. Ilya Sutskever, "Training Recurrent Neural Networks." PhD diss., University of Toronto, 2003, 10-11.

23. Alex Krizhevsky, Ilya Sutskever, and Geoffrey E Hinton, "ImageNet Classification with Deep Convolutional Neural Networks," Advances in Neural Information Processing Systems 25 (2012): 1097-1105.

24. Andreas Sudmann, "Wenn die Maschinen mit der Sprache spielen," Frankfurter Allgemeine Zeitung, November 2, 2016. However, a first demo version was not presented to the public until 2017. See https://www.youtube.com/ watch $? \mathrm{v}=7$ Fntm $8 \mathrm{cCbNM} \& \mathrm{t}=45$ s.

25. Later, in early summer 2019, TwentyBN started to develop its AI trainer for the fitness market. Accordingly, the company then focused on creating 
videos, showing different fitness exercises in order to train the AI model. The corresponding app called "Fitness Ally" was released exclusively on iOS in May 2020.

26. At the same, we have to acknowledge that AI's learning systems, even if they most certainly become more and more embodied as well as situated in our empirical world, nevertheless always remain an alien form of intelligence, very much different from the embodied intelligence of human beings.

27. Of course, this erasure of context is again a problematic ideological operation. In addition, the operations of the learning algorithms and in particular the learning data itself can be biased in many different respects. See Alexander Monea, "Race and Computer Vision," The Democratization of Artificial Intelligence: Net Politics in the Era of Learning Algorithms, ed. Andreas Sudmann (Bielefeld: Transcript, 2019), 189-208.

28. Sudmann, "Wenn die Maschinen mit der Sprache spielen."

29. Friedrich Kittler, "Die Künstliche Intelligenz des Weltkriegs: Alan Turing," in Friedrich Kittler and Georg-Christoph Tholen, ed., Arsenale der Seele. Literatur- und Medienanalyse seit 1870 (Munich: Fink, 1989), 187-202, 195.

30. Andreas Sudmann, "Szenarien des Postdigitalen. Deep Learning als MedienRevolution," in Machine Learning: Medien, Infrastrukturen und Technologien der Künstlichen Intelligenz, ed. Christoph Engemann und Andreas Sudmann, (Bielefeld: Transcript, 2018), 55-73.

31. At the same time, it is still important to note that ANN based systems operate as deterministic machines. See Sudmann, "Szenarien des Postdigitalen."

32. Friedrich Kittler, Grammophon, Film, Typewriter. (Berlin: Brinkmann und Bose, 1986).

33. Paul Virilio, La Machine de Vision (Paris: Galilée, 1988).

\section{About the Author}

Andreas Sudmann is a media scholar and AI researcher at Ruhr-University of Bochum, Germany. He is the author of several books, edited collections, and essays in the field of media and digital culture studies, including The Democratization of Artificial Intelligence. Net Politics in the Era of Learning Algorithms (Columbia University Press/ Transcript 2019), "Games and Artificial Intelligence" (Special Issue of Eludamos. Journal for Computer Game Culture Vol. 10 (2019), and "Artifıcial Intelligences" ("Künstliche Intelligenzen"), German Journal for Media Studies (Zeitschrift für Medienwissenschaft) Vol. 21 (2019). 